\title{
Regionale Verteilung der Cochlea-Implantat (CI)-versorgenden Einrichtungen in Deutschland
}

\section{Regional distribution of the cochlear implant $(\mathrm{Cl})$ centers in Germany}

Autoren

T. Stöver ${ }^{1}$, R. Zeh², B. Gängler², S. K. Plontke ${ }^{3}$, S. Ohligmacher², T. Deitmer4, O. Hupka², H. J. Welkoborsky ${ }^{5}$, M. Schulz², W. Delank ${ }^{6}$, M. Strobel ${ }^{2}$, O. Guntinas-Lichius ${ }^{7}$, S. Lang ${ }^{8}$, A. Dietz ${ }^{9}$, S. Dazert ${ }^{10}$, M. Diensthuber ${ }^{1}$

Institute

1 Klinik für Hals-Nasen-Ohrenheilkunde,

Universitätsklinikum Frankfurt a. M.

2 Deutsche Cochlea Implantat Gesellschaft (DCIG) e. V., Senden

3 Klinik für Hals-Nasen-Ohrenheilkunde, Kopf- und Halschirurgie, Universitätsklinikum Halle (Saale)

4 Deutsche Gesellschaft für Hals-Nasen-Ohrenheilkunde, Kopf- und Halschirurgie e. V., Bonn

5 HNO-Klinik, Klinikum Nordstadt, Hannover

6 HNO-Klinik, Klinikum der Stadt Ludwigshafen, Ludwigshafen

7 Klinik für Hals-Nasen-Ohrenheilkunde,

Universitätsklinikum Jena

8 Klinik für Hals-Nasen-Ohrenheilkunde, Universitätsklinikum Essen

9 Klinik für Hals-Nasen-Ohrenheilkunde, Universitätsklinikum Leipzig

10 Klinik für Hals-, Nasen- und Ohrenheilkunde, Kopfund Halschirurgie, St.-Elisabeth-Hospital, Klinikum der Ruhr-Universität Bochum

Schlüsselwörter

Cochlea-Implantat, regionale Verteilung, Deutschland, Cluster

Key words

cochlear implant, regional distribution, Germany, cluster

online publiziert 09.11 .2020

Bibliografie

Laryngo-Rhino-Otol 2020; 99: 863-871

DOI 10.1055/a-1302-0368

ISSN 0935-8943

(C) 2020. Thieme. All rights reserved.

Georg Thieme Verlag KG, Rüdigerstraße 14,

70469 Stuttgart, Germany

Korrespondenzadresse

Prof. Dr. Timo Stöver

Klinik für Hals-Nasen-Ohrenheilkunde

Universitätsklinikum Frankfurt a. M., Theodor-Stern-Kai 7, 60590 Frankfurt/Main, Deutschland

Tel.: $+49 / 69 / 63016788$

Fax: $+49 / 69 / 63015435$

timo.stoever@kgu.de
Dr. Roland Zeh

Deutsche Cochlea-Implantat-Gesellschaft (DCIG), Hauptstraße 43, 89250 Senden, Deutschland

Tel.: $+49 / 7307 / 9257474$

Fax: $+49 / 7307 / 9257475$

roland.zeh@dcig.de

\section{ZUSAMMENFASSUNG}

Die Versorgung von Patienten mit einer hochgradigen Schwerhörigkeit oder Taubheit mit einem Cochlea-Implantat (CI) stellt heute in Deutschland einen Standard dar. Umso überraschender ist es, dass kaum Daten zur aktuellen Anzahl der Cl-versorgenden Einrichtungen (Cl-Kliniken) und zu deren geografischer Verteilung existieren. Der Selbsthilfeverband Deutsche Cochlea Implantat Gesellschaft (DCIG) e. V. und die Deutsche Gesellschaft für Hals-Nasen-Ohrenheilkunde, Kopfund Halschirurgie (DGHNO-KHC) haben daher eine Umfrage zur Erfassung der regionalen Verteilung, des Leistungsspektrums, der Berücksichtigung existierender Qualitätsstandards sowie der Zusammenarbeit mit Patientenselbsthilfe-Organisationen der einzelnen $\mathrm{Cl}$-versorgenden Einrichtungen initiiert.

Hierfür wurden insgesamt 170 Kliniken für Hals-NasenOhrenheilkunde bzw. deren ärztliche Leiter (37 Lehrstuhlinhaber und 133 Chefärzte) per E-Mail kontaktiert und mittels Fragebogens befragt. Die Befragung erfolgte von Oktober 2019 bis Februar 2020.

Von den 170 kontaktierten Kliniken nahmen 71 (41,8\%) an der Umfrage teil. Hiervon bestätigten 70 Kliniken (98,6\%), Cl-Operationen durchzuführen. Damit gaben 41,8 \% aller kontaktierten Kliniken an, Cl-Operationen durchzuführen (70 von 170 Kliniken), während diese Information von 99 Kliniken nicht verfügbar war. Alle $70 \mathrm{Cl}$-versorgenden Einrichtungen (100\%) gaben an, Cl-Operationen an Erwachsenen, 60 Kliniken $(85,7 \%)$ ebenfalls bei Kindern (<18 Jahre) durchzuführen. 36 Kliniken (51,4\%) berichteten, dass die Gesamtanzahl der $\mathrm{Cl}$-Operationen an ihrer Einrichtung im Jahr 2018 mehr als 50 betrug. In 64 Kliniken (91,4\%) werden, nach den erhobenen Daten, die Empfehlungen der DGHNO-KHC zur CI-Versorgung (gemäß Weißbuch Cl-Versorgung, Stand 2018) berücksichtigt. Eine Zusammenarbeit der Klinik mit der Cl-Selbsthilfe wurde von 67 Kliniken (95,7\%) angegeben. Die geografische Verteilung der $\mathrm{Cl}$-versorgenden Einrichtungen, die an der 
Umfrage teilgenommen haben, zeigte ein heterogenes Verteilungsmuster zwischen den einzelnen Bundesländern und auch innerhalb der jeweiligen Bundesländer.

Die hier präsentierte Arbeit ist eine erste Standortbestimmung im Hinblick auf die regionale Verteilung der Cl-versorgenden Einrichtungen in Deutschland. Auffällig ist eine Clusterbildung der $\mathrm{Cl}$-Kliniken in Ballungsräumen, teils mit mehreren Einrichtungen in einer Stadt. Erfreulich ist die weit überwiegende Beachtung qualitätsbezogener Aspekte, wie die Berücksichtigung des CI-Weißbuchs der DGHNO-KHC und die Zusammenarbeit mit der Patientenselbsthilfe. Limitationen der Studie ergeben sich aus der begrenzten Teilnahme an der Umfrage (41,8\% der kontaktierten Kliniken).

\section{ABSTRACT}

The treatment of patients with severe hearing loss or deafness with a cochlear implant $(\mathrm{Cl})$ represents a standard in Germany today. However, there is hardly any data on the current number of clinical $\mathrm{Cl}$ centers ( $\mathrm{Cl}$ clinics) and their geographical distribution. The patient self-help organization, German Cochlear Implant Society (DCIG), and the German Society for Otorhinolaryngology, Head and Neck Surgery (DGHNO-KHC) have therefore initiated a survey to determine the regional distribution, the range of services, the consideration of existing quality standards and cooperation with patient self-help organizations of the individual clinical $\mathrm{Cl}$ centers.

For this purpose, a total number of 170 ENT departments or their directors (37 professors and 133 chief physicians), respectively, were contacted by e-mail and provided with a questionnaire. The survey took place from October 2019 to February 2020.

Of the 170 departments contacted, 71 (41.8\%) took part in the survey. Of these, 70 departments (98.6\%) confirmed to perform $\mathrm{Cl}$ surgeries. Thus, $41.8 \%$ of all clinics contacted reported to perform $\mathrm{Cl}$ surgeries (70 of 170 clinics), while this information was not available from 99 clinics. All 70 clinical $\mathrm{Cl}$ centers (100\%) reported to conduct $\mathrm{Cl}$ surgeries on adults, 60 centers $(85.7 \%$ ) also on children ( $<18$ years). 36 departments $(51.4 \%$ ) reported that the total number of $\mathrm{Cl}$ surgeries at their facility in 2018 was more than 50 . In 64 departments (91.4\%), the recommendations of the DGHNO-KHC on $\mathrm{Cl}$ care (according to the White Paper $\mathrm{Cl}$ care 2018) were followed. A collaboration between the department and patient self-help organization was confirmed by 67 institutions (95.7\%). The geographical distribution of the clinical $\mathrm{Cl}$ centers showed a heterogeneous distribution pattern between the individual federal states and also within the respective federal state.

The work presented here is a first assessment of the situation with regard to the regional distribution of clinical $\mathrm{Cl}$ centers in Germany. A clustering of $\mathrm{Cl}$ centers was noticeable in metropolitan areas, sometimes with several facilities in one city. The predominant attention to quality-related aspects, such as the consideration of the DGHNO-KHC white paper and the cooperation with patient self-help, is gratifying. The limitations of the study result from limited participation in the survey ( $41.8 \%$ of the contacted clinics).

\section{Einleitung}

Die Versorgung mit einem Cochlea-Implantat $(\mathrm{Cl})$ stellt heute für hochgradig schwerhörige oder gehörlose Kinder und Erwachsene den „Goldstandard“ einer Hörrehabilitation dar. Keine andere neuroprothetische Behandlung des Menschen hat in den letzten 30 Jahren eine vergleichbare Erfolgsgeschichte vorzuweisen. Für diese positive Entwicklung war eine Vielzahl unterschiedlicher Faktoren verantwortlich. Technische Weiterentwicklungen, wie die Einführung verbesserter Sprachverarbeitungsstrategien, die Entwicklung optimierter Elektrodendesigns, die Reduktion des Energieverbrauchs und die Entwicklung von HdO-Audioprozessoren, haben wesentlich zur Akzeptanz und zu verbesserten Ergebnissen beigetragen. Auch hat sich die Indikationsstellung zu einer Cl-Versorgung über die letzten 3 Jahrzehnte erheblich verändert. Während initial eine beidseitige komplette Taubheit vorliegen musste, wird heute eine frühzeitige Versorgung unter Erhaltung des vorhandenen Restgehörs angestrebt (elektrisch-akustische Stimulation, EAS). Inzwischen zählen auch die bilaterale Versorgung sowie die Versorgung einer einseitigen Taubheit bei gegenseitig normalem Gehör zum etablierten Standard. Gleiches gilt für die frühkindliche Versorgung innerhalb des ersten Lebensjahres und ebenfalls für die Versorgung ohne Altersbeschränkung im sehr hohen Lebensalter (Übersicht in [1]).
Mit der kontinuierlichen technischen Weiterentwicklung der $\mathrm{Cl}$, der Erweiterung der Indikationsstellung, dem hieraus resultierenden verbesserten Sprachverstehen und der gesteigerten Lebensqualität $\mathrm{Cl}$-versorgter Patienten hat sich die Anzahl der $\mathrm{Cl}$-Versorgungen in Deutschland seit der Einführung dieser Methode stark erhöht. Auch wenn es zur jährlichen Gesamtzahl der $\mathrm{Cl}$-Versorgungen in Deutschland keine absolut verlässliche Angabe gibt, kann zumindest über das Statistische Bundesamt die Auflistung der OPS-Kodierung der Kliniken erhoben werden [2]. Hiernach wurden im Jahr 2018 insgesamt 4441 Cl-Operationen dokumentiert. Diese Angabe umfasst ausschließlich vollstationäre Leistungserbringer und weist daher eine „Unschärfe“ auf. Dennoch gibt dieser Wert einen ungefähren Anhalt über die Größenordnung der jährlichen Versorgungen in Deutschland.

Eine ähnliche Unsicherheit existiert auch im Hinblick auf die aktuelle Anzahl der Cl-versorgenden Einrichtungen (Cl-Kliniken) und deren geografischer Verteilung in Deutschland. Bislang besteht kein umfassendes Verzeichnis, das Informationen zu diesem spezialisierten Leistungsangebot einer Einrichtung erheben würde. Dieser Umstand ist insbesondere für betroffene Patienten oder deren Angehörige unbefriedigend, da kein einfacher Zugang zu umfassenden Daten bezüglich der regionalen oder überregionalen Lokalisation von Einrichtungen existiert. Zur aktuellen Positionsbestimmung der $\mathrm{Cl}$-versorgenden Einrichtungen in 
Deutschland wurde die hier präsentierte Umfrage gemeinsam vom Dachverband der deutschen Cl-Selbsthilfeorganisationen, der Deutschen Cochlea Implantat Gesellschaft (DCIG) e. V. und der Deutschen Gesellschaft für Hals-Nasen-Ohrenheilkunde, Kopf- und Halschirurgie (DGHNO-KHC) initiiert. Ziel der Erhebung war die Erfassung der regionalen Verteilung der $\mathrm{Cl}$-versorgenden Einrichtungen, deren Leistungsspektrum, der Berücksichtigung existierender Qualitätsstandards sowie der Zusammenarbeit mit $\mathrm{Cl}$-Patientenselbsthilfe-Organisationen.

\section{Material und Methoden}

Die präsentierte Umfrage wurde durch die DCIG (Senden) mit Unterstützung der DGHNO-KHC (Bonn) durchgeführt. Hierfür wurden insgesamt 170 Kliniken für Hals-Nasen-Ohrenheilkunde bzw. deren ärztliche Leiter (37 Lehrstuhlinhaber und 133 Chefärzte) per E-Mail kontaktiert und ein Fragebogen (siehe unten) versendet. Die Aussendung erfolgte erstmals im Oktober 2019. Im Februar 2020 erfolgte die zweite Aussendung. Die Rücksendung des beantworteten Fragebogens erfolgte nicht anonym und wahlweise per E-Mail, Fax oder postalisch. Die letzte Antwort eines Teilnehmers ging am 22.02.2020 in der Geschäftsstelle der DCIG ein.

Insgesamt haben 71 Kliniken (41,8\%) an der Umfrage teilgenommen. Alle Teilnehmer, die bestätigten, Cochlea-Implantationen durchzuführen, haben ihr Einverständnis erklärt, dass die erhobenen Daten für die Erstellung einer Auflistung der Cl-versorgenden Einrichtungen („Cl-Klinik-Liste“) verwendet und publiziert werden dürfen.

Der versendete Fragebogen umfasste die nachfolgend aufgeführten Fragen. Die Fragen 1-4 und 6-7 konnten jeweils entweder mit „ja“, „nein“ oder „keine Angabe“ beantwortet werden. Frage 5 sollte mit einer Zahlenangabe beantwortet werden.

1. Führen Sie $\mathrm{Cl}$-Operationen durch?

2. Führen Sie Cl-Operationen bei Erwachsenen durch?

3. Führen Sie Cl-Operationen bei Kindern (<18 Jahren) durch?

4. War die Gesamtanzahl der $\mathrm{Cl}$-Operationen an Ihrer Einrichtung im Jahr 2018>50?

5. Wie groß ist die Anzahl der angebotenen Cl-Hersteller an Ihrer Einrichtung?

6. Werden die Empfehlungen der DGHNO-KHC zur Cl-Versorgung (gemäß Weißbuch Cl-Versorgung, Stand 2018) an Ihrer $\mathrm{Cl}$-versorgenden Einrichtung berücksichtigt?

7. Arbeiten Sie mit der örtlichen Cl-Selbsthilfe zusammen?

\section{Grafische Darstellung der Daten}

Die Erstellung der Abbildungen erfolgte mit GraphPadPrism 8 (GraphPadSoftware, San Diego, USA) und FreeHand MX (Adobe Inc., San Jose, USA).

\section{Ergebnisse}

\section{Fragebogenbasierte Datenerhebung}

Von den 170 kontaktierten Kliniken nahmen 71 (41,8\%) an der Umfrage teil. Die Ergebnisse der Befragung sind in Abb. 1 zusammengefasst. Insgesamt bestätigten 70 Kliniken (98,6\%), $\mathrm{Cl}$-Operationen durchzuführen ( $\mathbf{A} \mathbf{b b}$. 1a). Dies entspricht $41,8 \%$ aller kontaktierten Kliniken (70 von 170). Eine Klinik nahm zwar an der Umfrage teil, beantwortete jedoch nur die erste Frage ( $\triangleright$ Abb. 1a) und gab an, keine $\mathrm{Cl}$-Operationen durchzuführen. Von 99 Kliniken (58,2\%) ist nicht bekannt, ob dort Cl-Operationen durchgeführt werden.

Von den $\mathrm{Cl}$-versorgenden Einrichtungen gaben 70 Kliniken (100\%) an, Cl-Operationen an Erwachsenen durchzuführen ( $\triangleright$ Abb. 1b). Hingegen wurde von 60 Kliniken (85,7\%) angeführt, dass sie $\mathrm{Cl}$-Operationen ebenfalls bei Kindern (<18 Jahre) durchführen ( $\triangleright$ Abb. 1c). Keine Cl-Operation an Kindern wird an 8 Kliniken $(11,4 \%)$ durchgeführt. Keine Angabe hierzu wurde von 2 Kliniken $(2,9 \%)$ gemacht.

36 Kliniken $(51,4 \%)$ gaben an, dass die Gesamtanzahl der $\mathrm{Cl}$-Operationen an ihrer Einrichtung im Jahr 2018 mehr als 50 betrug. 25 Kliniken (35,7\%) gaben an, dass sie 201850 oder weniger $\mathrm{Cl}$-Operationen durchgeführt haben. Von 9 Kliniken (12,9\%) wurde hierzu keine Angabe gemacht ( $\vee$ Abb. 1 d).

Die Anzahl der angebotenen Implantate verschiedener Cl-Hersteller wurde von 32 Kliniken mit 4 (45,7\%), von 27 mit 3 (38,6\%), von 6 mit 2 (8,6\%) und von 4 mit 1 (5,7\%) Hersteller angegeben ( $\triangleright$ Abb. 1e). Eine Klinik $(1,4 \%)$ machte zu dieser Frage keine Angaben.

64 Kliniken (91,4\%) gaben an, die Empfehlungen der DGHNOKHC zur Cl-Versorgung (gemäß Weißbuch Cl-Versorgung, Stand 2018 [3]) zu berücksichtigen. Von einer Klinik wurde dies verneint $(1,4 \%)$ und von 5 Kliniken $(7,1 \%)$ wurde hierzu keine Angabe gemacht ( $\triangleright$ Abb. 1f).

Eine Zusammenarbeit der Klinik mit der Cl-Selbsthilfe wurde von 67 Kliniken (95,7\%) bestätigt. Keine Klinik (0\%) führte an, nicht mit der Cl-Selbsthilfe zusammenzuarbeiten. Keine Angabe hierzu erfolgte von 3 Kliniken (4,3\%) ( Abb. 1 g).

Die geografische Verteilung der $\mathrm{Cl}$-versorgenden Einrichtungen, die sich an der Umfrage beteiligt haben, zeigte ein heterogenes Verteilungsmuster zwischen den einzelnen Bundesländern und auch innerhalb des jeweiligen Bundeslandes ( $\triangleright$ Abb. 2, $\triangleright$ Tab. 1).

\section{Diskussion}

Die Versorgung schwerhöriger oder ertaubter Patienten mit einem Cochlea-Implantat $(\mathrm{Cl})$ hat in den vergangenen 3 Jahrzehnten eine beeindruckende Entwicklung genommen. Die verbesserten Erfolge der mit einem Cl versorgten Patienten, das zunehmende Wissen um die Existenz und die Effektivität der Methode innerhalb der Ärzteschaft und auch die Erweiterung der Indikationen haben inzwischen zu einer weiten Verbreitung dieser Behandlung geführt. War die Cl-Versorgung in den ersten Jahren nur auf wenige Einrichtungen beschränkt, hat sie sich inzwischen an vielen Orten innerhalb Deutschlands etabliert. 
A

Führen Sie Cl-Operationen durch?

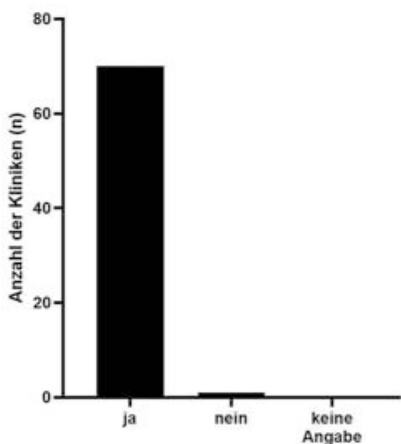

D War die Gesamtanzahl der
Cl-Operationen an Ihrer
Einrichtung im Jahr $2018>50 ?$

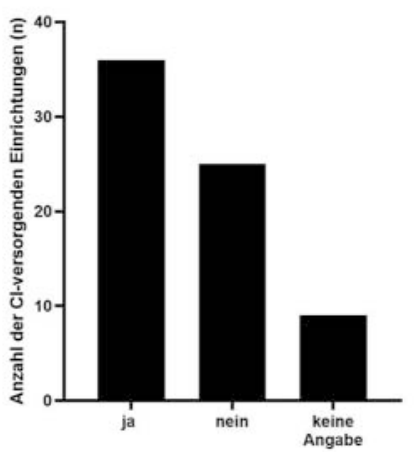

G

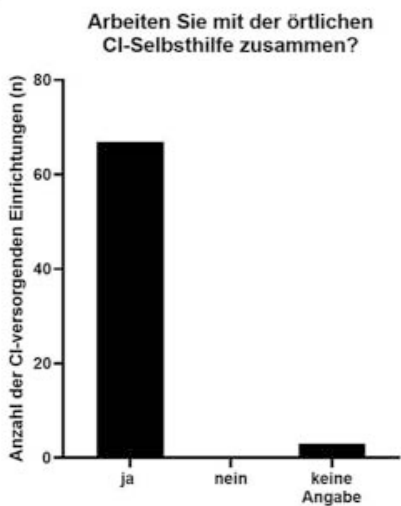

B

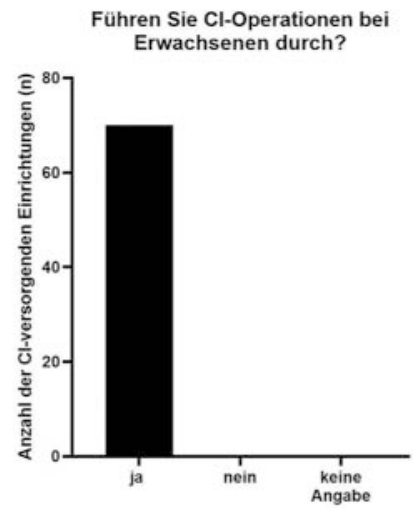

E
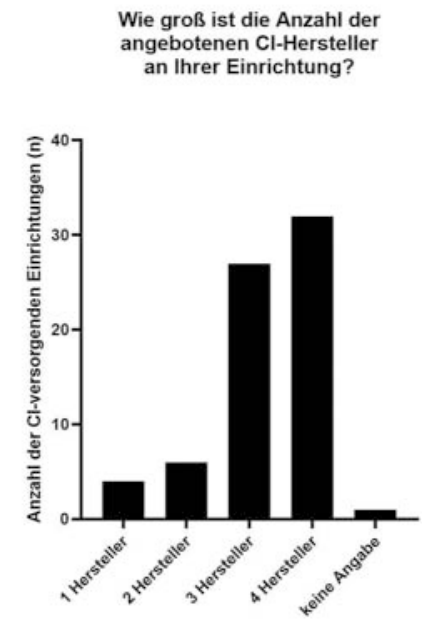

C

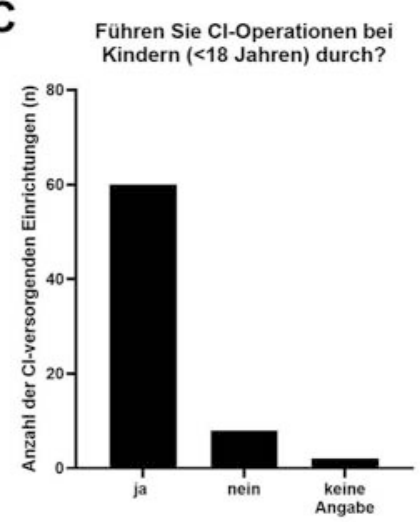

$\mathbf{F}$

Werden die Empfehlungen der DGHNOKHC zur Cl-Versorgung (gemäß Weißbuch Cl-Versorgung, Stand 2018)

an Ihrer $\mathrm{Cl}$-versorgenden Einrichtung berücksichtigt?

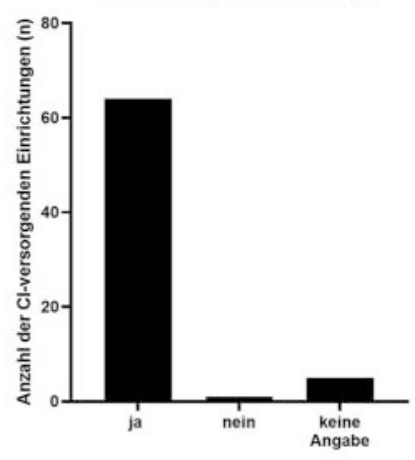

- Abb. 1 Ergebnisse der Umfrage unter Lehrstuhlinhabern und Chefärzten in Deutschland. Die in A-G genannten Fragen wurden von den Leitern der Kliniken ( $n=71$ für $\mathbf{A}, \mathbf{n}=70$ für $\mathbf{B}-\mathbf{G}$ ) entweder mit „ja“, „nein“ oder „keine Angabe“ A-D, F-G bzw. mit einer Zahlenangabe oder „keine Angabe“ E beantwortet. Eine Klinik verneinte die Durchführung einer Cl-Versorgung A und nahm folglich an der Beantwortung der Fragen in B-G nicht teil. 


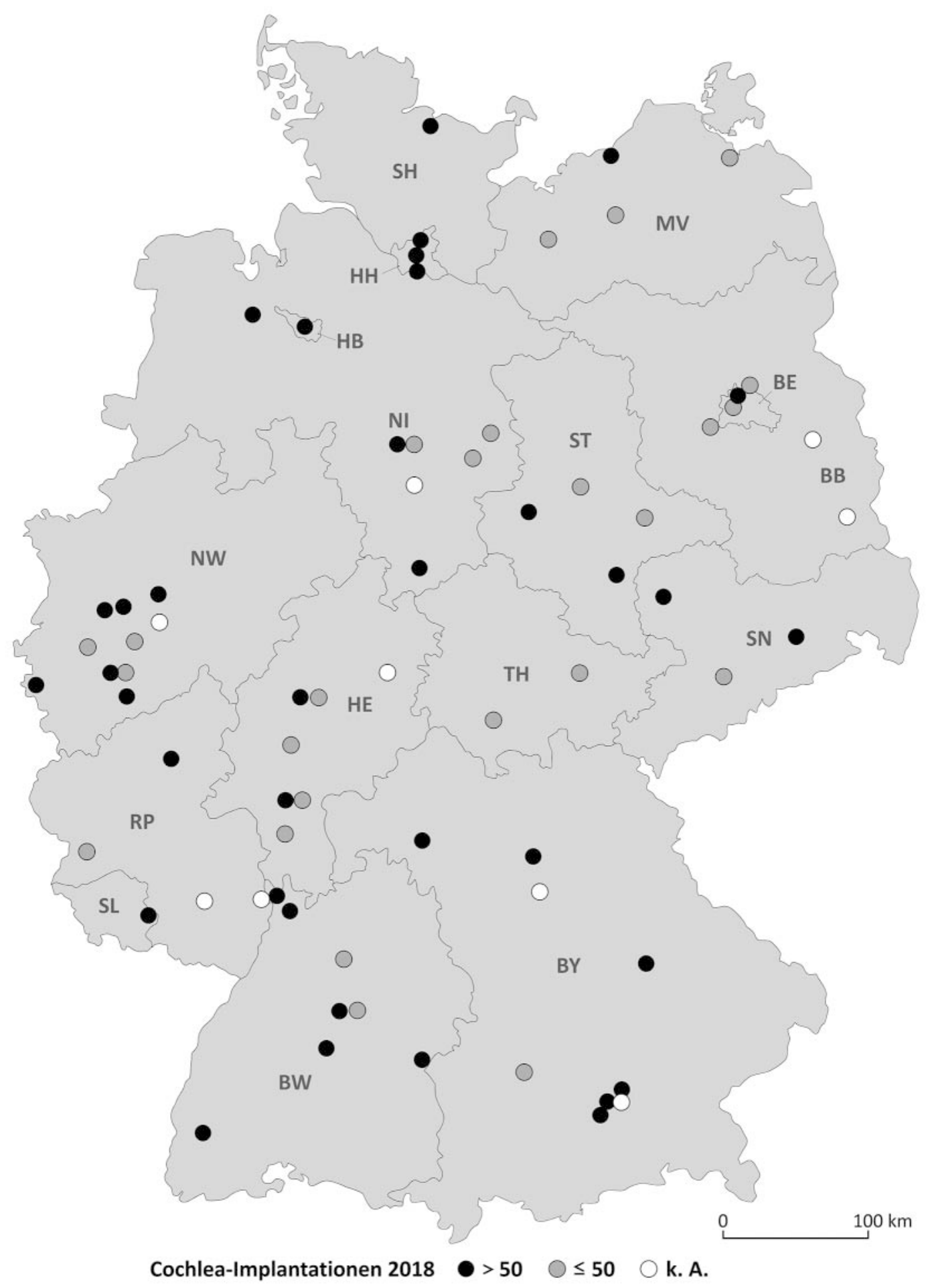

- Abb. 2 Verteilung der Cl-versorgenden Einrichtungen in Deutschland. Aufgeführt sind Kliniken, die im Jahr $2018>50$ (schwarzer Punkt; $\mathrm{n}=36$ ) Cochlea-Implantationen durchführten, Kliniken, die im Jahr $2018 \leq 50$ Cochlea-Implantationen durchführten (grauer Punkt; $n=25$ ) sowie Kliniken, die hierzu keine Angabe machten (weißer Punkt; $n=9$ ). Abkürzungen: keine Angabe (k. A.), Baden-Württemberg (BW), Bayern (BY), Berlin (BE), Brandenburg (BB), Bremen (HB), Hamburg (HH), Hessen (HE), Mecklenburg-Vorpommern (MV), Niedersachsen (NI), Nordrhein-Westfalen (NW), Rheinland-Pfalz (RP), Saarland (SL), Sachsen (SN), Sachsen-Anhalt (ST), Schleswig-Holstein (SH), Thüringen (TH). 

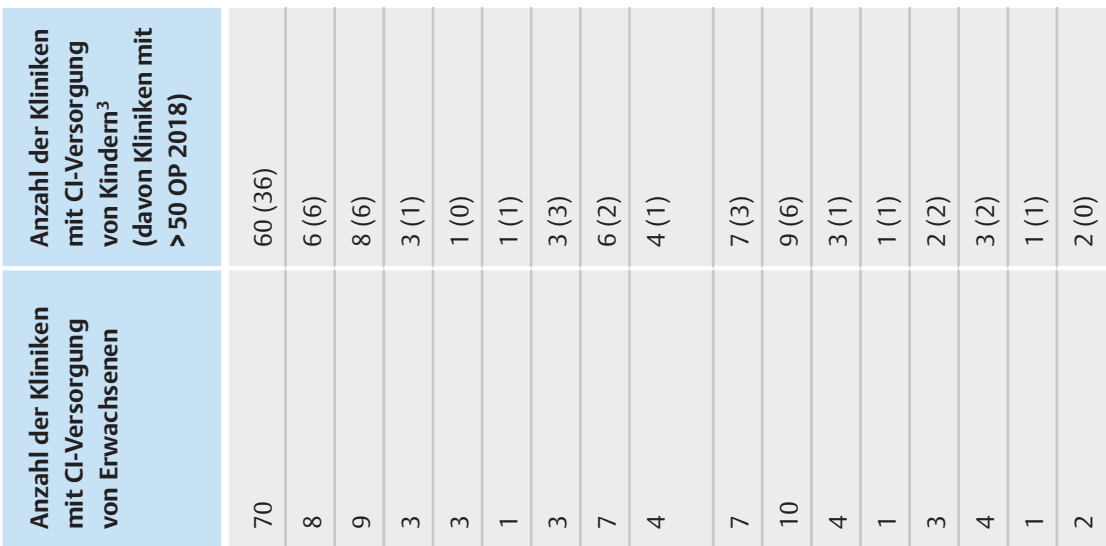

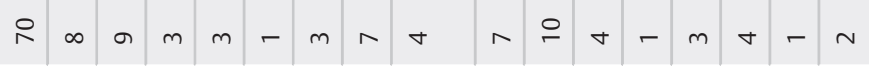

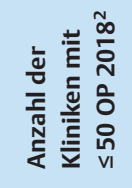

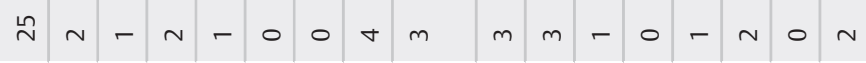
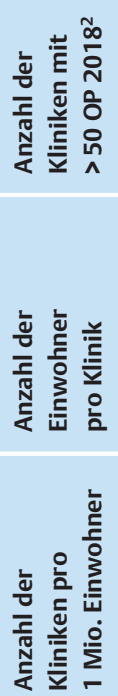

$\stackrel{m}{0} 0-0-m n-m b--n N-0$

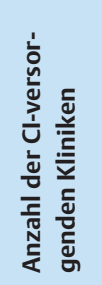

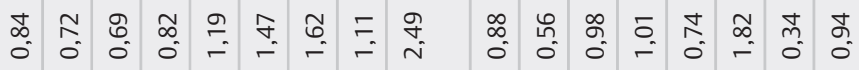

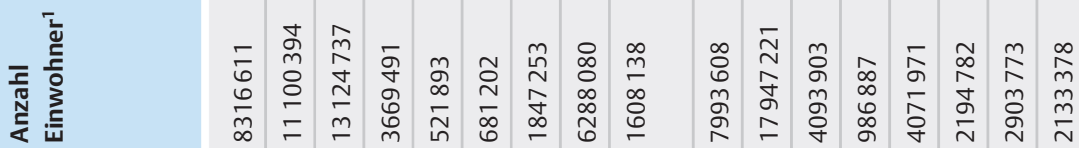

$\therefore \infty a m m-m h a r o r-m a-n$

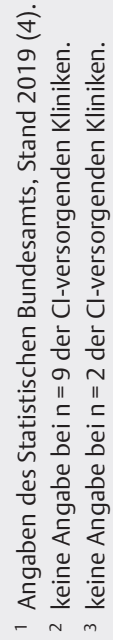


Bislang existierten keine allgemein verfügbaren Daten zur regionalen Verteilung der $\mathrm{Cl}$-versorgenden Einrichtungen oder zum jeweiligen $\mathrm{Cl}$-bezogenen Leistungsspektrum einer Klinik. Die DCIG und die DGHNO-KHC haben daher erstmals eine Erhebung der regionalen Verteilung, des Leistungsspektrums, der Berücksichtigung existierender Qualitätsstandards sowie der lokalen Zusammenarbeit mit Patientenselbsthilfe-Organisationen durchgeführt. Die erhobenen Daten erlauben zudem eine Reihe von Rückschlüssen über die geografische Verteilung der Cl-versorgenden Einrichtungen in Deutschland.

Insgesamt haben ca. $40 \%$ (70 der 170) der kontaktierten Kliniken in der Umfrage eine Cl-Versorgung an der jeweiligen Einrichtung bestätigt. Aus jedem der 16 Bundesländer hat zumindest eine $\mathrm{Cl}$-Klinik an der Umfrage teilgenommen und bestätigt, dass von ihr eine $\mathrm{Cl}$-Versorgung durchgeführt wird. Die erhobenen Daten ermöglichen damit auch eine Darstellung der Cl-Versorgung über die gesamte Fläche Deutschlands.

Die regionale Verteilung auf die einzelnen Bundesländer zeigt erhebliche Unterschiede und variiert zwischen je einer Klinik in Bremen, in Schleswig-Holstein sowie im Saarland und 10 Kliniken in Nordrhein-Westfalen. Die Verteilung der 70 Kliniken auf die Bundesländer wird in $>$ Tab. 1 und in $>$ Abb. $\mathbf{2}$ dargestellt. Da sich aber nicht nur die Fläche der Bundesländer, sondern auch deren Bevölkerungszahl stark voneinander unterscheiden, wurde die Anzahl der Cl-Kliniken in Beziehung zur jeweiligen Bevölkerungszahl des Bundeslandes [4] gesetzt ( $\triangleright$ Tab. 1). Aus den erhobenen Daten wurde für ganz Deutschland eine durchschnittliche Anzahl von 0,84 Cl-Kliniken pro 1 Million (Mio.) Einwohner bestimmt (70 Cl-Kliniken bei ca. 83 Millionen Einwohnern). Dies entspricht ca. 1,2 Mio. zu versorgende Einwohner pro Cl-Klinik ( $\vee$ Tab. 1). Die regionalen Unterschiede variieren wiederum stark im Vergleich zu diesem Durchschnittswert. Während in Schleswig-Holstein mit 0,34 Cl-Kliniken pro 1 Mio. Einwohner (entspricht 2,9 Mio. Einwohner pro Klinik) der Minimalwert vorliegt, findet sich der Maximalwert in Mecklenburg-Vorpommern mit 2,49 Cl-Kliniken pro 1 Mio. Einwohner (entspricht ca. 0,4 Mio. Einwohner pro Klinik) ( $\triangleright$ Tab. 1).

Die Detailbetrachtung der „Cl-Deutschlandkarte“ ( $>$ Abb. 2 ) offenbart eine Reihe von bemerkenswerten Besonderheiten. Zunächst wird deutlich, dass die regionale Lokalisation und Verteilung der Kliniken nicht homogen in der Fläche eines Bundeslandes vorliegen. Dies ist auch nicht unbedingt zu erwarten, denn mehrheitlich handelt es sich bei der Übernahme der Cl-Versorgung durch eine Klinik nicht um das Resultat einer Versorgungsplanung, sondern ist (vermutlich) eher der fachlichen Schwerpunktbildung einer HNO-Klinik, der Entscheidung der HNO-ärztlichen Leitung oder auch der ökonomischen Entscheidung einer Krankenhausadministration geschuldet. Eine „Leistungsplanung“ einer übergeordneten Institution, wie des Gesundheitsministeriums des Bundeslandes, liegt der regionalen Verteilung dieser Kliniken (wahrscheinlich) nicht zugrunde.

Eine homogene geografische Verteilung der Cl-Kliniken ist auch deshalb nicht unbedingt zu erwarten, da auch die Bevölkerung innerhalb eines Bundeslandes nicht homogen in der Fläche verteilt ist und deutlich mehr Menschen in den (städtischen) Ballungsräumen einer Region leben. Dies zeigt sich auch im Verteilungsmuster der Cl-Kliniken in der Cl-Landkarte ( $\bullet$ Abb. 2). Ein besonderes Beispiel ist der „Rhein-Ruhr-Cluster“, der über das Ruhrgebiet entlang des Rheins mehrere Kliniken in einem flächenmäßig kleinen, aber bevölkerungsreichen Teil Deutschlands konzentriert. Aus dieser Situation ergibt sich ein scheinbares Paradoxon, dass viele Menschen in einer kleinen Region von vielen Einrichtungen versorgt werden, die Kliniken zum Teil aber nur wenige Kilometer voneinander entfernt sind. Eine ähnliche Clusterbildung findet sich im Rhein-Main-Neckar-Raum.

Ein weiteres interessantes Verteilungsphänomen zeigt sich in Bezug auf viele Großstädte, die z. T. mehrere Cl-Einrichtungen aufweisen. Hierzu zählen u. a. Berlin, Hamburg, München, Frankfurt, Hannover oder Köln. Die Bewertung einer Clusterbildung stellt eine komplexe Herausforderung dar. Zu berücksichtigen ist hierbei im Hinblick auf eine Interpretation dieser Daten wiederum die Anzahl der Menschen, die in einer Stadt oder einem Ballungsraum leben und versorgt werden. Dies gilt insbesondere, da innerhalb einer Großstadt oder Clusterregion, z. B. „Rhein-Ruhr“, eine hohe Bevölkerungsdichte existiert, die anhand von Durchschnittswerten des jeweiligen Bundeslandes nur ungenügend dargestellt wird. Ebenfalls ist gerade in Ballungsräumen der Übergang zwischen 2 oder auch mehreren Städten fließend, sodass eine klare Zuordnung der Einwohner zu einer Klinik, zumindest anhand der hier erhobenen Daten, kaum möglich ist.

Die Beurteilung der Verteilung der Cl-versorgenden Einrichtungen in einem Bundesland stellt auch aus einem weiteren Grund eine Herausforderung dar: Im Rahmen dieser Studie wurde allein erfasst, ob eine Klinik die Cl-Operation durchführt. Nicht direkt erhoben wurde, ob und in welchem Umfang eine Basis- oder Folgetherapie/Cl-Rehabilitation bzw. Nachsorge angeboten wird. Für eine erfolgreiche Hörrehabilitation ist die Durchführung dieser Maßnahmen unerlässlich und muss zur Beurteilung der Versorgungsqualität perspektivisch ebenso erhoben werden. Die weitaus überwiegende Mehrzahl der Cl-Kliniken gab an, dass die Empfehlungen des „Cl-Weißbuchs“ [3] berücksichtigt werden und damit auch die Empfehlung zur Durchführung einer entsprechenden technischen, hörsprachtherapeutischen, audiologischen und medizinischen Betreuung des Patienten sowie ggf. die Durchführung einer Cl-Rehabilitation. Die „Qualität“ der lokalen Struktur zur Durchführung der Cl-Rehabilitation wurde aber nicht direkt erhoben, sodass keine abschließende Aussage zu diesem Bereich anhand der hier verfügbaren Daten möglich ist. Da dies allerdings nicht das Ziel der hier präsentierten Studie war, wird dies Gegenstand zukünftiger Untersuchungen werden.

Anhand der im Rahmen dieser Umfrage erhobenen Daten soll keine Aussage im Hinblick auf eine „Über- oder Unterversorgung“ einer Region mit Cl-versorgenden Einrichtungen getroffen werden. Dies ist weder Ziel der Arbeit, noch lassen die erhobenen Daten hierauf ausreichend Rückschlüsse zu. Ergänzend kann auch festgestellt werden, dass der Umfang der Tätigkeit einer Klinik im Bereich der $\mathrm{Cl}$-Versorgung deutlich variiert. Das Spektrum der diesbezüglichen Anzahl an Eingriffen variiert zwischen einigen wenigen und mehreren hundert $\mathrm{Cl}$-Operationen pro Jahr innerhalb einer Klinik [5]. Ein indirekter Hinweis auf die große Diversität des Leistungsumfangs der Kliniken ergibt sich auch aus den hier erhobenen Daten: 36 Kliniken gaben an, dass sie mehr als $50 \mathrm{Cl}$-Operationen pro Jahr durchführen. Dies entspricht etwa der Hälfte aller Einrichtungen, die eine $\mathrm{Cl}$-Versorgung bestätigt haben. 
Die Anzahl der durchgeführten Eingriffe ist zweifelsohne kein Parameter, der allein die Qualität einer Versorgung bestimmt. Besondere Berücksichtigung sollte die Beachtung der existierenden Qualitätsstandards finden (Übersicht in [6]). Hier sind besonders das „Weißbuch Cochlea-Implantat-Versorgung in Deutschland“ der DGHNO-KHC [3] und die derzeit in Überarbeitung befindliche $\mathrm{Cl}$-Leitlinie [7] zu nennen. Diesbezüglich ist es erfreulich, dass mehr als $90 \%$ der Cl-Kliniken angeben, dass sie die Empfehlungen der DGHNO-KHC zur Cl-Versorgung (gemäß Weißbuch Cl-Versorgung, Stand 2018 [3]) berücksichtigen.

Die im „Cl-Weißbuch“ [3] benannten Qualitätskriterien richten sich auch auf die Patienteninteressen im Hinblick auf eine umfassende Beratung zur Cl-Versorgung und die Option, sich zwischen verschiedenen Cochlea-Implantat-Systemen entscheiden zu können. Auch hier ergeben die Umfrageergebnisse ein sehr positives Bild des existierenden Qualitätsstandards. Fast 85 \% der Kliniken bieten mindestens 3 Implantate unterschiedlicher Hersteller an. Im Vergleich zu der bis vor wenigen Jahren durchaus üblichen „Monokultur“, d. h. Cl-Implantate nur eines einzigen Herstellers vorzuhalten, ist dies sicher eine bemerkenswert positive Entwicklung, die für die konsequente Umsetzung der Qualitätsempfehlungen der DGHNO-KHC durch die Kliniken spricht.

Die Cl-Versorgung ist ein komplexer Vorgang, in dem die Patienten-Selbsthilfe eine besondere Rolle einnimmt. Eine Zusammenarbeit zwischen Kliniken und Cl-Selbsthilfe wird daher auch durch das CI-Weißbuch [3] empfohlen, und Vertreter der Selbsthilfe nehmen auch an Beratungen zur Erstellung wissenschaftlicher Leitlinien teil [7]. Es ist daher aus der Patientenperspektive sehr positiv zu bewerten, dass über $95 \%$ der Kliniken mit der $\mathrm{Cl}$-Selbsthilfe zusammenarbeiten. Die Umsetzung dieses Aspekts durch nahezu alle Cl-Kliniken ist als ein Zeichen einer hohen patientenbezogenen Versorgungsqualität zu werten.

In Bezug auf die Cl-Versorgung von Erwachsenen und Kindern findet sich ein differenziertes Bild. Während alle 70 Kliniken eine Versorgung von Erwachsenen angeben, bestätigen dies etwa $85 \%$ auch für die Cl-Versorgung von Kindern. In der überwiegenden Anzahl der Einrichtungen erfolgt damit sowohl eine Versorgung von Erwachsenen und Kindern. Von den 60 Kliniken, die sowohl eine Erwachsenen- als auch eine Kinderversorgung vornehmen, geben 36 Kliniken an, dass sie mehr als 50 Cl-Operationen pro Jahr (2018) durchführten. Hieraus folgt, dass 24 Kliniken, die eine $\mathrm{Cl}$-Versorgung bei Kindern durchführen, weniger als $50 \mathrm{Cl}$-Operationen durchführten oder hierzu keine Angaben machten.

Wie bereits zuvor erwähnt liegt die Zielsetzung dieser Studie nicht in der Diskussion der Frage einer Mindestmenge in der $\mathrm{Cl}$-Versorgung. Hierzu bieten die im Rahmen dieser Studie erhobenen Daten keinen rationalen Ansatz zur Beurteilung der Effekte einer Anzahl von Cl-Operationen auf die resultierende Versorgungsqualität. Daher kann auch kein Rückschluss aus der hier erfassten Anzahl von 50 Cl-Operationen pro Jahr (2018) bezüglich einer ausreichenden oder nicht ausreichenden Qualität in der Cl-Versorgung abgeleitet werden, da diese Grenzzahl rein arbiträr gewählt wurde. Dennoch erscheint es bemerkenswert, dass häufig eine große räumliche Nähe zwischen Kliniken mit mehr oder weniger als $50 \mathrm{Cl}$-Operationen pro Jahr besteht ( $\triangleright$ Abb. 2).
Die im Rahmen dieser Untersuchung erhobenen Daten zeigen, dass die Empfehlungen des Weißbuchs Cl-Versorgung [3] von der großen Mehrheit der Kliniken im Hinblick auf die Zusammenarbeit mit Patientenselbsthilfe-Organisationen und das Angebot verschiedener Implantate umgesetzt werden. Klare Unterschiede zeigen sich in der Anzahl der durchgeführten Cl-Operationen und der regionalen Verteilung, mit einer Clusterbildung in vielen Städten und Ballungsräumen. Im Hinblick auf die Bewertung müssen medizinisch-fachliche von politischen Perspektiven unterschieden werden. Auch wenn diese Untersuchung zeigt, dass die empfohlenen Qualitätsstandards offensichtlich weit überwiegend eingehalten werden, existieren derzeit erhebliche Aktivitäten zur Umsetzung einer Leistungsplanung im Cl-Bereich. Als Beispiel kann hier Nordrhein-Westfalen genannt werden, das eine Strukturierung der Krankenhäuser nach Leistungsgruppen und Leistungsbereichen plant. Durch die NRW-Krankenhausplan-Initiative könnte sich neben der Onkologie auch die Cl-Versorgung zu einem Modellbereich zur Leistungsplanung innerhalb eines Bundeslandes mit Festlegung von Leistungserbringern und Leistungsumfang entwickeln. Dieser Ansatz bietet zugleich Chancen und Risiken für die großen Maximalversorgungsthemen der stationären Leistungserbringung der HNO-Heilkunde. Es bleibt abzuwarten, ob diese landespolitische Initiative tatsächlich über eine Fokussierung der Ressourcen eine weitere Verbesserung der Versorgungsqualität bewirkt oder eher der Kosteneinsparung und Rationalisierung der Leistungserbringung dienen wird.

\section{Limitationen der Studie}

An der hier präsentierten Umfrage haben 71 von 170 kontaktierten Kliniken teilgenommen. Die Bewertung der Umfrageergebnisse ist daher immer vor dem Hintergrund einer relativ geringen Teilnahmequote an der Erhebung von 41,8\% zu betrachten.

70 von 170 der kontaktierten Kliniken haben in Form einer Selbstauskunft angegeben, dass sie eine Cl-Versorgung durchführen. Lediglich eine Klinik gab an, keine $\mathrm{Cl}$-Versorgung anzubieten. Hinsichtlich der $\mathrm{Cl}$-Versorgung an den verbleibenden 99 Kliniken ist keine abschließende Aussage möglich, da keine Rückmeldung gegeben wurde. Objektive Daten zur Gesamtzahl der Cl-Kliniken in Deutschland existieren derzeit nicht bzw. sind nicht einfach zugänglich. Gegenwärtig sind am ehesten Abrechnungsdaten der Kostenträger hilfreich, um die Gesamtzahl zu bestimmen. Nach Angaben der Techniker Krankenkasse wurden im Jahr 2018 allein bei dieser Krankenkasse von 93 Einrichtungen in Deutschland entweder die DRG D01A oder D01B (Cochlea-Implantation einseitig und beidseitig) zur Abrechnung gebracht (persönliche Kommunikation G. Lehmann, Techniker Krankenkasse Hamburg). Hieraus ergibt sich, dass mindestens 23 Kliniken nicht an der Umfrage teilgenommen haben, obwohl sie eine Cl-Versorgung durchführen.

Da diese Zahlenangaben ausschließlich die Angaben der Techniker Krankenkasse repräsentieren, könnten durchaus auch mehr als $93 \mathrm{Cl}$-Kliniken in Deutschland existieren. Berücksichtigt werden muss hierbei, dass in Einzelfällen auch eine Cl-Versorgung an nichtstationären Einrichtungen stattfinden könnte. Da im Rahmen dieser Studie lediglich stationäre Einrichtungen (Kliniken) 
angeschrieben wurden, könnte damit die tatsächliche Zahl der $\mathrm{Cl}$-versorgenden Einrichtungen in Deutschland sogar bei mehr als 100 liegen.

Die Betrachtung der Anzahl der Cl-Kliniken pro Bundesland bzw. pro Einwohner des jeweiligen Bundeslandes ist aufgrund der derzeit nicht zugänglichen Daten der Einrichtungen, die sich nicht an der Umfrage beteiligt haben, erheblichen Unsicherheiten ausgesetzt. Gerade durch die gegenwärtig scheinbar geringe oder durchschnittliche Anzahl von Cl-Kliniken in einzelnen Bundesländern würde es durch die Integration weiterer Datensätze bislang nicht beteiligter Kliniken zu deutlichen Veränderungen der Ergebnisse kommen. Allein aus dieser Betrachtung heraus ist anhand der derzeit verfügbaren Daten eine Bewertung der Dichte der Cl-Kliniken nicht abschließend möglich. Dennoch lassen sich, wie in der Diskussion ausgeführt, bereits anhand der aktuell verfügbaren Daten „Clusterregionen“ und Trends für die regionale Verteilung der Cl-Kliniken identifizieren.

Die hier erhobenen Daten beruhen auf der Eigeneinschätzung und Rückmeldungen der angeschriebenen Kliniken. Eine unabhängige Verifikation der Informationen auf Korrektheit der gemachten Angaben konnte nicht erfolgen. Abweichungen der Angaben sind damit zumindest möglich. Allerdings existiert gegenwärtig auch keine andere einheitliche, verifizierte Datenquelle, die eine Überprüfung der Angaben erlauben würde.

\section{Schlussfolgerung}

Die hier präsentierte Arbeit ist eine erste Standortbestimmung im Hinblick auf die regionale Verteilung der $\mathrm{Cl}$-versorgenden Einrichtungen in Deutschland. Die Ergebnisse zeigen eine heterogene regionale Verteilung der Anzahl und Lage der Kliniken in den einzelnen Bundesländern. Auffällig ist eine Clusterbildung der Cl-Kliniken in Ballungsräumen, teils mit mehreren Einrichtungen in einer Stadt. Erfreulich ist die weit überwiegende Berücksichtigung qualitätsbezogener Aspekte wie die Beachtung des Cl-Weißbuchs der DGHNO-KHC (3) und die Zusammenarbeit mit der Patientenselbsthilfe. Die erhobenen Daten bieten zudem Hinweise, dass sich die Datenqualität durch ein deutschlandweites Cl-Register weiter verbessern ließe. Hierzu könnte das von der DGHNO-KHC in Vorbereitung befindliche $\mathrm{Cl}$-Register einen erfolgversprechenden Lösungsansatz bieten.

Interessenkonflikt

Die Autorinnen/Autoren geben an, dass kein Interessenkonflikt besteht.

Danksagung

Die Autoren danken Herrn Wolfgang Fraedrich und Frau Miriam Blumenstock für die Unterstützung bei der grafischen Darstellung der Daten sowie Frau Ulrike Fischer für die administrative Unterstützung.

\section{Literatur}

[1] Dazert S, Thomas JP, Loth A et al. Cochlear implantation diagnosis, indications, and auditory rehabilitation results. Dtsch Arztebl Int 2020; 117: $690-700$

[2] Statistisches Bundesamt. Krankenhäuser. https://www.destatis.de/DE/ Themen/Gesellschaft-Umwelt/Gesundheit/Krankenhaeuser/_inhalt. html\#sprg369934. Stand: 19.10.2020

[3] Deutsche Gesellschaft für Hals-Nasen-Ohren-Heilkunde, Kopf- und HalsChirurgie. Weißbuch Cochlea-Implantat(Cl)-Versorgung. https://cdn. hno.org/media/PDF/ci-weissbuch-und-register-dghno-1-auflage-stand04-2018.pdf. Stand: 29.10 .2020

[4] Statistisches Bundesamt. Bevölkerung nach Bundesländern. https://www.destatis.de/DE/Themen/Gesellschaft-Umwelt/Bevoelkerung/ Bevoelkerungsstand/Tabellen/bevoelkerung-nichtdeutsch-laender.html. Stand: 19.10 .2020

[5] Verband der Universitätsklinika Deutschlands (VUD). Zahlen und Fakten 2015. https://www.uniklinika.de/fileadmin/user_upload/pdf/Qualit_7_.pdf. Stand: 30.10 .2020

[6] Stöver T, Leinung M, Loth A. Welche Qualität macht den Unterschied in der Cochlea-Implantat-Versorgung? Laryngorhinootologie 2020; 99 (Suppl. 1): S107-S164

[7] AWMF. Leitlinie Cochlea-Implantat Versorgung einschließlich zentralauditorischer Implantate. https://www.awmf.org/leitlinien/detail/II/ 017-071.html. Stand: 19.10.2020 